\title{
Solar Energetic particles at pileup collisions of the multiple-shock
}

\author{
Xin Wang ${ }^{* \dagger}$ \\ Xinjiang Astronomical Observatory, Chinese Academy of Sciences, Urumqi 830011, China \\ Key Laboratory of Radio Astronomy, Chinese Academy of Sciences, Nanjing 210008, China \\ E-mail: wangxinexao.ac.cn
}

\section{Yihua Yan}

CAS Key Laboratory of Solar Activity, NAOC, Beijing 100012, China

\section{Mingde Ding}

Key Laboratory of Modern Astronomy and Astrophysics (Nanjing University), Ministry of Education, Nanjing 210093, China

\begin{abstract}
The ground-level enhancement (GLE) events are often associated with the large gradual solar events such as fast coronal mass ejections (CMEs), but not all fast CMEs lead to GLE events. We suggest that GLE would be associated with processes of the multiple CMEs interactions. Here we proposed a "multiple-shock" scenario driven by the "continuous-CME" sympathetically, in which the posterior shocks catch up with the preceding shock and have pileup collisions. We simulate the "multiple-shock" by applying a dynamic Monte Carlo method in present work. In the "multipleshock" scenario, the seed energetic particles produced by the preceding shock can be injected into the posterior shocks for cascaded acceleration efficiently. We obtain the detailed SEP energy spectrum with different behaviors at the related episodes of the "multiple-shock" evolution. As results, we find the pileup collisions of the "multiple-shock" dominate multiple“concave" energy spectral slope in the generated SEP event.
\end{abstract}

36th International Cosmic Ray Conference -ICRC2019-

July 24th - August 1st, 2019

Madison, WI, U.S.A.

\footnotetext{
* Speaker.

${ }^{\dagger}$ This work is supported by the Xinjiang Natural Science Foundation No. 2019D01A100. Part supported by Xinjiang Heaven Lake Hundred-Talent Program, and NSFC (No.11673056).
} 


\section{Introduction}

Two key questions regarding SEPs within a Space Weather framework are: Which solar events are responsible for the production of SEPs most dangerous for Space Weather? What type of events can give rise to extreme SEP enhancements[26]? In this paper we provide modeling efforts related to SEPs, with the aim of addressing the above questions.

Impulsive SEP events are short for less than one day, low intensity and numerous with the average number about 1000/year. Gradual events are long for several days at energies of a few $\mathrm{MeV} / \mathrm{nuc}$, rather rare for decades per year, and orders of magnitude more intense in protons than impulsive SEP events. The gradual events, characterized by the largest proton fluxes and therefore of most relevance to Space Weather, are ascribed to acceleration by CME-driven shocks as they propagate through the heliosphere. In gradual events, protons can be accelerated into the $\mathrm{GeV}$ range, and, when directed towards the Earth, may lead to neutron monitors (NMs) detecting events at the Earth's surface. These GLEs are the most extreme of solar events, and thus are of special interest to the heliophysics community [1,37]. Our understanding of energetic solar events and specifically GLEs increased dramatically during solar cycle 23 [20] as a result of advances in instrumentation and an abundance of events to observe. Solar cycle 24, being much quieter than the previous one, has so far provided only two unambiguous GLEs: GLE71 on May 17th 2012 and GLE72 on September 10th 2017. GLE events are one type of large gradual SEP event. These events can be observed by ionization chambers and NMs on the ground. In these events, protons and ions are accelerated to very high energies with intensities often 10-100 times larger than normal gradual SEP events. In solar cycle 23, there were 16 GLEs and the last one occurred on 2006 December 13. The observed particle time intensity profiles of GLEs are similar to those of normal gradual SEP events. This suggests that the underlying acceleration of GLE events is the same as that operating in normal gradual SEP events - the diffusive shock acceleration (DSA) [16, 9]. The standard DSA mechanism was born in 1970s [2, 27, 4, 7]. Its application in the interplanetary shock can CME-driven shocks have been taken by various authors. Lee [28, 29] solved the coupled particle transport and wave action equations. The approach provided a self-consistent formalism for evaluating Alfvén wave amplification and particle energization at a quasi-parallel shock. Using the steady state solution of the coupled system, Gordon et al. [21] examined particle acceleration at the Earth's bow shock. This formalism is extended by Lee [29], who considered explicitly a two stream approximation of the energetic particles. $\mathrm{Ng}$ et al. [38] used the same equations as Lee [28, 29], but solved the time dependent wave transport equation. Such an approach allows one to obtain the wave acceleration and energetic particle spectrum in a time dependent manner. This can be potentially important in studying the early phases of GLE events. Zank et al. [47] and Li et al. [32, 33], on the other hand, assumed instantaneous steady-state solution of the DSA, and included the time consideration by balancing the shock dynamic time with the acceleration time. Using this approach, Li et al. [34] examined the spectral break behavior in large SEP events and argued that the spectral breaks that often observed in observation must be $\mathrm{Q} / \mathrm{A}$ dependent and the dependence is due to the shock geometry.

For investigating why do GLEs have larger intensity and higher energies than the normal gradual SEPs? Li et al. [30] proposed a "twin-CME" scenario for GLE events and large SEP events upon solar cycle 23. In this scenario, two CMEs go off closely in time from the same active region (AR). 
The preceding CME drives a shock which generates a very turbulent downstream and produces the seed of the normal energetic particles. As the posterior CME plunges into the strong turbulent region downstream of the preceding shock, it will accelerate them to very high energies[11, 19]. Depending on whether there is reconnection between the magnetic field turbulence occurred on the posterior CME-driven shock and that produced by the preceding CME, the pre-accelerated ions inside the preceding CME's driver can be processed by the posterior CME, leading to an enhancement of ions that are compositionally interplanetary CME-like[31]. For further extending this study, we proposed a "multiple-shock" scenario to investigate the GLEs production and its energy spectral behavior in details.

\section{Model}

In the late 1970s, many authors [2, 27, 4, 7] introduced the test-particle theory of particle acceleration at strong collisionless shocks due to the first-order Fermi mechanism. However, quantitative estimates soon pointed out that this DSA mechanism may be so efficient that the backreaction of the accelerated particles on the shock dynamics cannot be neglected. The obvious theoretical challenge is how to model effectively the full shock dynamics. Modelling shocks with efficient particle acceleration using plasma simulations [particle-in-cell (PIC) and hybrid] is extremely computationally expensive for several reasons. First, the energies of charged particles participating in the process range from the low thermal energies of cold plasma to the ultrarelativistic energies of cosmic rays (CRs), and both extremes of particle spectra are dynamically important if acceleration is efficient (see e.g. Vladimirov et al. [41], for estimates of computational requirements). Secondly, simulations need to be done in three dimensions because of the possibility of non-physical suppression of important processes in one- and two-dimensional simulations (see e.g. Jones et al. [22]). Therefore, approximate methods must be used to model efficient particle accelerating shocks.

This relevant piece of physics has been taken into account by adopting a number of rather different approaches, namely (i) fully numerical simulations, in which a time-dependent diffusionconvection equation for the CR transport is solved in concert with coupled gas-dynamic conservation laws, like in Bell [5], Berezhko \& Völk [6], Kang \& Jones [24], Zirakashvili \& Aharonian [48]; (ii) stationary Monte Carlo numerical simulations of the full particle population, like in Ellison et al. [15, 16], Ellison, Baring \& Jones [13], Ellison \& Double [14], Vladimirov et al. [41] and dynamical Monte Carlo numerical simulations of the full particle population, like in Knerr et al. [25], Wang et al. [43, 42, 46]. (iii) semi-analytic solutions of the stationary (or quasi-stationary; see Blasi et al. [8] diffusion-convection equation coupled to the gas-dynamic equations, like in Malkov et al. [36], Caprioli et al. [10]. All these approaches make consistent predictions about the main consequences of the shock modification, i.e. the appearance of an upstream precursor created by the CR pressure around the shock, which slows down the incoming fluid. As a consequence of this CR-induced velocity gradient, the fluid compression ratio is no longer 4 for any strong shock. Hence, the test-particle prediction that the spectrum of accelerated particles has to be $\propto p^{4}$ is no longer valid. Nevertheless, it is still true that the spectral slope mainly depends on the compression factor actually "felt" by CRs. Since the larger is its momentum, the farther from the shock a particle can diffuse, high (low) momentum particles probe a total compression ratio larger (smaller) than 4. The resulting CR spectrum becomes rather concave, being harder (softer) than $p^{4}$ at the 
higher (lower) energies. For accurate reviews of CR-modified shocks, the reader can refer to Jones \& Ellison [23], Malkov \& Drury [35].

In Monte Carlo simulations, one follows particles scattering off the magnetic irregularities based on an assumed scattering law. As the background flow around a one-dimensional shock which is assumed to be in a steady-state, Ellison et al. [16] used steady-state Monte Carlo method to calculate the particle spectra accelerated in the parallel component of Earth's bow-shock and successfully compared them with observational data. They showed that the agreement between simulation results and observed data was quite impressive. But the highest energy accelerated by the shock only goes up to $100 \mathrm{keV}$ due to the small size of Earth's bow-shock. They also showed that the results of Monte Carlo simulations were consistent with those of hybrid plasma simulations. Baring et al. [3] also did the same kind of comparison with the observed data in oblique interplanetary shocks and also came up with excellent agreements. Later Knerr et al. [25] developed a dynamically time-dependent Monte-carlo simulation for the Earth's bow-shock, and give the production of the more than $4 \mathrm{MeV}$ energetic particles at the high energy "tail".

In an effort to complement and extend such studies, we focus on the impact of the pileup shocks on the multiple-shock wave evolution and propagation. So a dynamical Monte Carlo model for the study of twin parallel collisionless shocks and their associated particle acceleration is developed. Our multiple-shock model using the dynamical Monte Carlo code means that the angular momentum diffusive behavior is based on a prescribed assumption obeying a certain distribution in the scattering process. Under the isotropic scattering angular distribution, we can readily follow particles as they move about the shock and scatter in the background flow. In this isotropic scattering model, particle injection and escape are treated in a natural, self-consistent manner.

This multiple-shock Monte Carlo simulation presented here employ the prescribed isotropic scattering angular distributions based on earlier dynamical simulations done by Knerr et al. [25] to study Earth's parallel bow shocks. Since the pitch angle scattering law models particle scattering off the collective fields of the plasma, calculation of the electric and magnetic fields is unnecessary and is omitted. Under the assumption of the isotropic scattering angular distributions algorithm, particles scatter off the infinitely massive scattering centers elastically with a random angle between 0 and $\theta_{\max }$ in their local flow frame. In addition, we assume a constant scattering time (i.e., the mean time between two scattering events) for all particles, which implies particles' mean free paths are proportional to velocity. This idea that such a simple law can be used to describe the entire scattering process was postulated by Eichler [12], based on the two-stream instabilities work done by Parker [39]. Put simply, it is assumed that the turbulence generated by both energetic particles streaming in front of the shock and by thermal particles produces nearly elastic scattering for particles of all energies in diffusive shocks.

\section{Multiple-shock Simulation}

This model describes the "multiple-shock" interactions of a large SEP event at the interplanetary space within $7.5 R_{\odot}$, which would trigger off the related GLE event at the Earth surface. This means that there are multiple distinct CMEs with the same propagation direction in this event. According to the hight of the four CMEs at different times, the hight $h_{1}=6.68 R_{\odot}$ for CME1, the hight 


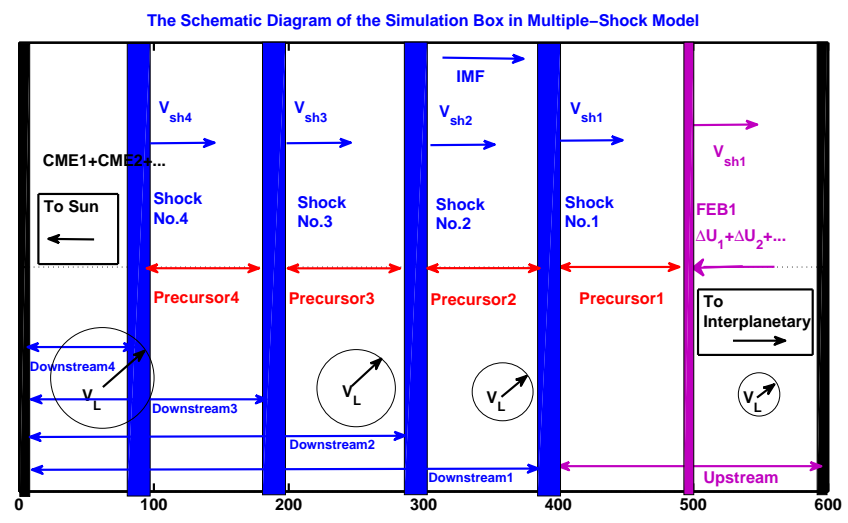

Figure 1: A schematic diagram of the multiple-shock simulation box. The left reflective wall represents the CMEs produce shocks No.1, No.2, No.3 and No.4 (representing by four blue vertical bars ) propagating from the left boundary to the right of the simulation box. After a period of time, the CME1 produce shock No.1 propagating into the simulation box, then the CME2, CME3 and CME4 also produces shock No.2, No.3, and No.4 appearing at the left boundary of the simulation box continuously. The multiple shocks evolve into the simulation box with a pileup interaction.

$h_{2}=5.01 R_{\odot}$ for CME2, the hight $h_{3}=3.34 R_{\odot}$ for CME3 and the hight $h_{2}=1.67 R_{\odot}$ for CME4 at the different time are given. The difference of the hight between the two CMEs is $1.67 R_{\odot}$.

Fig. 1 shows a schematic diagram of the "multiple-shock" model. The left reflective wall represents four CMEs and produce the shocks at the different time. Shocks No.1, No.2, No.3 and No.4 propagate from the left boundary of the simulation box to the right boundary. After the shock No.1 propagating into the inner of the simulation box with a relative bulk speed of $\Delta U_{1}$, the shock No. 2 begins to propagate from the left reflective wall to pursue the shock No.1 with a relative bulk speed $\Delta U_{2}$, and then CME3 and CME4 also produce shock No.3 and No.4 consequently. The four blue vertical bars at the simulation box indicate the shock No.1, No.2, No.3 and No.4 fronts, respectively. Initially, the upstream bulk flow speed of the shock No.1 is $\Delta U_{1}$ and the downstream bulk flow speed of shock No.1 is zero at the rest shock No.1 reference frame. When the shock No.2 entering into the simulation box, then two shocks begin to have an interaction each other with a relative speed of $\Delta U_{2}$. Once the rest shock No.1 reference frame turns to the rest shock No.2 reference frame, the upstream and downstream bulk speeds of the shock No.1 should be added an increment of the bulk speed $\Delta U_{2}$ becoming into the $\Delta U_{1}+\Delta U_{2}$ and $\Delta U_{2}$, respectively. The upstream and downstream bulk speeds of the shock No.2 correspondingly become the $\Delta U_{2}$ and zero, respectively. The shock No.3 and shock No.4 is the same order. In the "multiple-shock" scenario, since there exists a relative bulk speed $\Delta U$ between the two sympathetic shocks, the downstream bulk flow of the shock No.1 is always compressed by the shock No.2 unless the shock No. 2 catches up with and exceeds the shock No.1. So the heating and accelerated ions inside the downstream of the shock No.1 can be as the seed of the energetic particles for re-accelerating by the Fermi mechanism at the shock No.2. The shock No.3 and shock No.4 also obey the same rule. We apply a dynamic Monte Carlo technique to simulate this "multiple-shock" scenario in detail for investigating the integral energy spectral characters.

We consider four quasi-parallel shocks related with a large SEP event where the supersonic 
flows move from Sun to the interplanetary along the $x$-axis direction. With the preceding shock propagating from Sun along the $x$-axis to the interplanetary. The posterior shock propagates along the same direction. In order to investigating the enhancement intensity of this associated GLE event, we use a particle simulation method to reconstruct the energy spectrum of the related SEP event. Here, we apply a nonlinear dynamic Monte Carlo code to simulate "multiple-shock" scenario containing the back-reactions of the accelerated particles on the upstream sub-shock in front of the four shocks, respectively. In this model, the shock No.1 initially produces the heating and accelerated ions as the seed energetic particles for further acceleration when they penetrate into the the shock No.2. The shock No.2 moves forward to the shock No.1 with a relative bulk speed $\Delta U_{2}$ with the same direction alone $x$ axis. According to the ordinary observations, we can take the initial upstream bulk speed with the value for $U_{0}==400 \mathrm{kms}^{-1}$, the relative bulk speed of the shock No.1 with a value for $\Delta U_{1}=400 \mathrm{kms}^{-1}$ between its upstream and downstream bulk flows, the relative bulk speed of the shock No.2 with a value for $\Delta U_{2}=400 \mathrm{kms}^{-1}$ between its upstream and downstream bulk flows, the relative bulk speed of the shock No.3 with a value for $\Delta U_{3}=400 \mathrm{kms}^{-1}$ between its upstream and downstream bulk flows, and the relative bulk speed of the shock No.4 with a value for $\Delta U_{4}=400 \mathrm{kms}^{-1}$ between its upstream and downstream bulk flows. Note here, the downstream of the shock No.1 is also the upstream of the shock No.2. The shock No.3 and No.4 is also the same rule.

In this Monte Carlo method, we apply an initial number density of particles $n_{0}$ in the upstream bulk flow of the shock No.1, which obeys a Maxwellian distribution with a thermal speed $v_{L 0}$. The shock No.1 remains the relative bulk speed $\Delta U_{1}$ between the upstream and downstream bulk flow till the shock No.2 appears at the left boundary of the simulation box. The shock No.3 and shock No.4 is also the same rule. The total simulation time is $t_{\max }$. After a period of the simulation time, we start the shock No.2 to pursue the shock No.1 from the left boundary of the box with a relative speed $\Delta U_{2}$. During the next period of the simulation time, the double shocks undergo a pileup collision leading to the magnetic field amplification and the energetic ions' re-acceleration efficiently. As the new shock entering into the simulation box, the rest shock No.2 reference frame insteads of the rest shock No.1 reference frame. At the new rest shock reference frame, both the old upstream and downstream bulk speeds of the shock No.1 accordingly obtain an increment of the bulk speed $\Delta U_{2}$, respectively; The upstream and downstream bulk speeds of the shock No.2 are becoming the $\Delta U_{2}$ and zero, respectively. The shock No.3 and shock No.4 obey the same rule. Whatever the rest shock reference frame, the background solar wind bulk speed still hold on the value of $U_{0}$. Initially, we suggest the shock No.1 as a single-CME driven shock produces a normal power-law energy spectrum at a period of the total simulation time. Then at the beginning of the next period of the total simulation time, the shock No.2 driven by CME2 begins to catch up with the shock NO.1 and dramatically modify the existing normal power-law energy spectrum inside the downstream region of the shock No.1. The additional modifications provided by the shock No. 2 would bring some new variations on the old energy spectrum produced by the shock No.1. But in this "multiple-shock" scenario, since the posterior CME-driven shock catches up with the preceding CME-driven shock and have a pileup collision at the same direction when they propagate forward into the interplanetary space, the positive effect of the "multiple-shock" on the energetic particles would enhance the existing energy spectrum. We predict there probably appears multiple "concave" energy spectrum at a certain energy range on the related SEP event. 
The scattering process is usually modeled by some dependencies of the mean free path $\lambda_{m f p}$ of the particles on momentum $p$ in most particle simulation cases. Thus, in order for the acceleration to be efficient, a large number of shock crossings back and forth on the shock front are required. Shock particle acceleration therefore depends on the scattering process which is clearly a stochastic process, depending on the presence of scattering centers upstream and downstream and on the random changes in the scattering angle. It assumes that the scattering is elastic and isotropic for conserving energy. This mechanism always works until the gyroradius of the accelerated particle becomes so large that it exceeds the size of the system or the energy of the particle becomes so large that the back-scattering is ineffective. In order to experience the first scattering, the particle must initially already possess a gyroradius much larger than the entire width of the shock transition region. Only when this condition is satisfied, the shock will behave like a slightly thin discontinuity separating two regions of very different bulk speeds. A particle crossing back and forth over such shock can become aware of the bulk difference in speed and gain energy additions. In fact, for entering into the Fermi acceleration mechanism a particle must be pre-heated until its gyroradius becomes larger than the width of the shock [40].

Monte Carlo method applies a scattering law for particle diffusive processes on shocked plasmas, and the details of the scattering process are described in Wang et al. [43, 44, 42]. We assume that the particles scatter elastically off the background scattering centers with their scattering angles obeying an isotropic distribution in their local frame. In this scattering scenario, the assumption of elastic scattering requires that scattering centers are frozen into the background fluid; Simultaneously, the assumption of a constant collision time for all particles requires the particle's mean free path is proportional to its local velocity in the local frame[17]. For simplicity, we take its formula as

$$
\lambda_{m f p} \propto v_{L}
$$

For pitch-angle scattering, if the time in the local frame required for the particle momentum vector to accumulate deflections of the order of $90^{\circ}$ is identified with the collision time $\tau=\lambda_{m f p} / v_{L}$, where $v_{L}$ is the particle speed in the local frame, it was shown in the above references that the maximum deflection a particle experiences in an interaction satisfies

$$
\delta \theta_{\max }=\sqrt{6 \delta t / \tau}
$$

where $\delta t$ is the time in the local frame between pitch-angle scatterings. The stochastic scattering process is simulated with two random numbers, $\varphi$ and $\delta \theta$. At each time step $\delta t$, the azimuthal angle $\varphi$ is chosen randomly between 0 and $2 \pi$, and the particle pitch angle, $\delta \theta$, is chosen from a uniform distribution of $\cos \delta \theta$ between 1 and $\cos \delta \theta_{\max }$ [18]. The average number of scattering events occurring in an increment of time-step $\delta t$ depends on the average collision time $\tau$, and the scattering rate is presented by

$$
R_{s}=\delta t / \tau
$$

where $R_{s}$ is the probability of the scattering events occurring in an increment of time-step. The candidates with their local velocities and scattering angles scatter off the grid-based scattering centers. These individual particles do not change their routes until they are selected to scatter once again. For the individual protons, the grid-based scattering center can be seen as a sum of individual momenta. So these scattering processes can be taken as the elastic collisions. In an 
increment of time-step, once all of the candidates complete these elastic collisions, the momentum of the grid-based scattering center is changed. The total simulation temporally evolves forward by repeating the time-step sequence. To calculate the scattering processes accurately and produce an exponential mean free path distribution, the time-step should be much less than the collision time (i.e. $\delta t<\tau$ ). In these diffusive processes, the particles in the upstream region transfer their kinetic energy into their random thermal energy in the downstream region. Then a few of random thermal particles can be injected from the downstream region into the upstream region by multiple scattering cycles on shock fronts to obtain more energy gains and become the energetic particles.

Table 1: The Simulation Parameters

\begin{tabular}{|l|c|c|}
\hline Physical Parameters & Dimensionless Values & Scaled Values \\
\hline Upstream bulk speed 0 & $U_{0}=0.3$ & $400 \mathrm{kms}^{-1}$ \\
Relative upstream bulk speed 1 & $\Delta U_{1}=U_{1}-U_{0}=0.3$ & $400 \mathrm{kms}^{-1}$ \\
Relative upstream bulk speed 2 & $\Delta U_{2}=U_{2}-U_{1}=0.3$ & $400 \mathrm{kms}^{-1}$ \\
Relative upstream bulk speed 3 & $\Delta U_{3}=U_{3}-U_{2}=0.3$ & $400 \mathrm{kms}^{-1}$ \\
Relative upstream bulk speed 4 & $\Delta U_{4}=U_{4}-U_{3}=0.3$ & $400 \mathrm{kms}^{-1}$ \\
Initial thermal velocity & $v_{\mathrm{L}}=0.02$ & $26.9 \mathrm{kms}^{-1}$ \\
Scattering time & $\tau_{0}=0.833$ & $7.15 \mathrm{~s}$ \\
Box size & $X_{\max }=600$ & $10 R_{\odot}$ \\
Total time & $t_{\max }=2400$ & $343 \mathrm{munites}$ \\
Time step size & $d t=1 / 15$ & $0.57 \mathrm{~s}$ \\
Number of zones & $m_{\mathrm{x}}=1200$ & $\ldots$ \\
Initial particles per cell & $n_{0}=300$ & $\ldots$ \\
\hline
\end{tabular}

Notes: The $R_{\odot}$ is the radii of the Sun. The scale factors for distance, velocity, and time are $X_{\text {scale }}=10 R_{\odot} / 600$, $U_{\text {scale }}=400 \mathrm{kms}^{-1} / 0.3$, and $t_{\text {scale }}=X_{\text {scale }} / U_{\text {scale }}$. The dimensionless values and the scaled values can be transformed by the scaled factors each other.

In this multiple-shock simulation box, all the simulated parameters are listed in Table 1, According to the observations, we adjust the observed parameters for applying the appropriate simulated parameters. We present the scaled values of the parameters as follows. The background solar wind bulk speed is $U_{0}=400 \mathrm{kms}^{-1}$; The bulk speed of the CME1 is $U_{1}=800 \mathrm{kms}^{-1}$; The bulk speed of the CME2 is $U_{2}=1200 \mathrm{kms}^{-1}$; The bulk speed of the CME3 is $U_{3}=1600 \mathrm{kms}^{-1}$; The bulk speed of the CME4 is $U_{4}=2000 \mathrm{kms}^{-1}$; We define the relative upstream bulk flow speed of the shock No.1 is $\Delta U_{1}=U_{1}-U_{0}=400 \mathrm{kms}^{-1}$; the relative upstream bulk flow speed of the shock No.2 is $\Delta U_{2}=U_{2}-U_{1}=400 \mathrm{kms}^{-1}$; the relative upstream bulk flow speed of the shock No.3 is $\Delta U_{3}=U_{3}-$ $U_{2}=400 \mathrm{kms}^{-1}$; the relative upstream bulk flow speed of the shock No.4 is $\Delta U_{4}=U_{4}-U_{3}=400 \mathrm{kms}^{-1}$; The initial local thermal velocity is $v_{L 0}=26.9 \mathrm{kms}^{-1}$. The scattering time is $\tau=7.15$ seconds. The box size is chosen to be the $X_{\max }=10 \mathrm{R}_{\odot}$ for ensuring the "multiple-shock" interaction within the box (where $\mathrm{R}_{\odot}$ is the solar radius, about 109 times of the Earth radius $\mathrm{R}_{e}$ ). The total time of the simulation is chosen to be $t_{\max }=343$ minutes and long enough for producing the enhancements of the SEP event. Accordingly the time step is set to be $\delta t=0.57$ seconds.

The above scaled values of the parameters are corresponded to the follow dimensionless parameters, respectively. The relative upstream bulk flow speed of each shock $\Delta U_{1}=\Delta U_{2}=\Delta U_{3}=$ $\Delta U_{4}=0.3$; Initial local thermal velocity $v_{L 0}=0.02$; The constant of the collision time $\tau=0.833$; The total size of the box $X_{\max }=600$; The total simulation time $t_{\max }=2400$; The time step $\delta t=1 / 15$. 
These dimensionless values can be scaled by the distance $(X)$, time $(t)$, and velocity $(U)$ scaling factors: $X_{\text {scale }}=10 R_{\odot} / 600, U_{\text {scale }}=400 \mathrm{kms}^{-1} / 0.3$, and $t_{\text {scale }}=X_{\text {scale }} / U_{\text {scale }}$, respectively. In addition, we give the simulation box grids of $m_{x}=1200$, and the initial density of particles in each grid is $n_{0}=300$. The total number of the particles in the simulation box at the end of the simulation archives to more than one million particles.

\section{Energy Spectra}

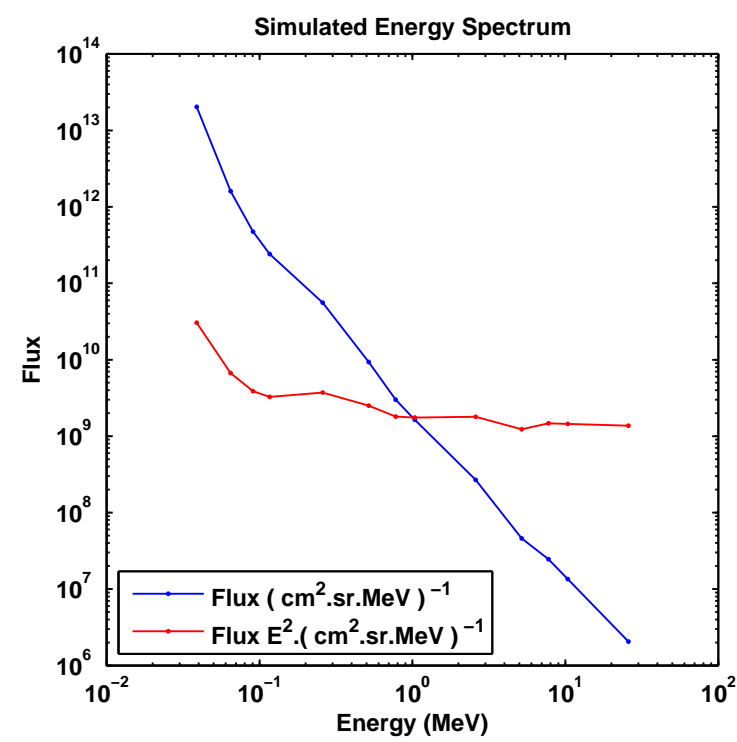

Figure 2: The simulated energy spectrum calculated from the multiple-shock scenario. The blue curve represents the particle fluxes with the normal representation, the red curve represents the energy spectrum with a specific representation by using a factor of the square of the energy times the flux.

Fig. 2 shows the simulated energy spectrum with two different representations for particle fluxes. The blue curve represents the particle fluxes with the normal representation, the red curve represents the energy spectrum with a specific representation by using a factor of the square of the energy times the flux. From the Fig.2, the energy spectrum covers the total energy range up to $20 \mathrm{MeV}$. The suprethermal particle "tail" is shown at the energy range less than $\sim 100 \mathrm{keV}$. At the energy range from the $100 \mathrm{keV}$ to $\sim 2 \mathrm{MeV}$, the energy spectrum represented by red curve shows a few lower "concave" shapes and some upper "bump" shapes. The three upper "bumps" are located in $200 \mathrm{keV}, 2 \mathrm{MeV}$, and $\sim 8 \mathrm{MeV}$ of the energy axis, respectively. The three lower "concave" points are located in $100 \mathrm{keV}, 1 \mathrm{MeV}$, and $5 \mathrm{MeV}$ of the energy axis. At the energy range from $100 \mathrm{keV}$ to $20 \mathrm{MeV}$, the soft energy spectrum show its index more than 2; the hard energy spectrum shows its index less than 2 . The red curve can clearly show that "concave" and "bump" properties in the total energy spectrum. According to our calculation from the red curve, we predict that these "concave" and "bump" points are associated with the multiple shock pileup point interaction. 


\section{Summaries and Conclusions}

In summary, we simulate the multiple-shock system for predicting the proton spectrum directly. We obtain the total energy spectrum covering the energy range up to $20 \mathrm{MeV}$. We also find the simulated energy spectrum exhibits the energy spectral "concave" and "bump" points at the energy axis. Comparably, we have ever investigated an energy spectrum "break" at $\sim 5.5 \mathrm{MeV}$ in our previous converging double-shock model. So, why do the converging shocks in previous study would produce a "broken" energy spectrum and the "pileup" multiple-shock in present study would produce a "concave" and "bump" properties in energy spectrum? There would be follow reasons: (i) According to the diffusive shock acceleration theory, the acceleration efficient is determined by the diffusive coefficient[42]. The attainable highest energy particle is depended on the diffusive length of particles scaled by the size of the precursor region[43]. At the converging shocks, the precursor region size will be shorten and fewer and fewer high energy particles gain energies resulting a softer energy at the high energy "tail"[46]. (ii) But in the multiple-shock scenario, the multiple-shock interaction can extend the precursor region size and enhance the existing accelerated particle distribution. The pre-accelerated particles caused by the first shock, penetrate into the posterior shocks for re-accelerating and modifying the existing power-law multiply to become a complicated energy spectrum. These processes can lead to a"concave" and "bump" shape on the energy spectrum. (iii) Furthermore, whatever in the converging shocks and the multiple-shock model, both the diffusive particles can extend the energy spectrum up to a few decades of $\mathrm{MeV}$ in their high energy "tails". The specific energy spectral shape would be depended on the type of the collision, in which the important parameter of the precursor size will be decided.

\section{References}

[1] Asvestari, E., Willamo, T., Gil, A., \& et al. 2017, Adv. Sp. Res., , 60, 781

[2] Axford, W. I., E. Leer, \& G. Skadron (1977), in Proc. 15th Int. Comsmic Ray Conf. (Plovdiv), 1978, Volume 11, p132-137

[3] Baring, M. G., Ellison, D. C. \& Jones, F. C., 1995, Adv. Sp. Res., , 15, 397

[4] Bell, A. R., 1978, MNRAS, 182, 147

[5] Bell A. R., 1987, MNRAS, 225, 61

[6] Berezhko E. G., \& Völk H. J., 1997, Astropart. Phys., , 7, 183

[7] Blandford, R. D., \& J. P. Ostriker, 1978, ApJ221, L29

[8] Blasi, P., Amato, E. \& Caprioli, D., 2007, MNRAS375,1471

[9] Bykov, A. M., Ellison, D. C, Osipov, S. M., \& Vladimirov, A. E. 2014, ApJ, 789 , 137

[10] Caprioli, D., Blasi, P. \& Amato, E., 2010, Astropart. Phys., 33, 307-311

[11] Ding, L. G., Li, G., Jiang, Y., Le, G., \& et al. 2014, ApJ, 793, L35

[12] Eichler, D., 1979, ApJ, 229, 419 
[13] Ellison, D. C., Baring, M. G. \& Jones, F. C., 1996, ApJ, 473, 1029

[14] Ellison, D. C. \& Double, G. P., 2002, Astropart. Phys., , 18, 213

[15] Ellison, D. C. \& Eichler, D., 1984, ApJ,286, 691

[16] Ellison, D. C., Möbius, E. \& Paschmann, G. 1990, ApJ, 352, 376

[17] Ellsion, D. C., Warren, D. C. \& Bykov, A. M. 2016, MNRAS, 456, 3090

[18] Ellsion, D. C., Warren, D. C. \& Bykov, A. M. 2013, ApJ, 776, 46

[19] Gopalswamy, N., Xie, H., Akiyama, S., et al. 2013, ApJL,765, L30

[20] Gopalswamy, N., Xie, H., Yashiro, S., et al. 2012, Space Sci. Rev. , 171, 23

[21] Gordon, B. E. \& et al., 1999, J. Geophys. Res., 104, 28263

[22] Jones F. C., Jokipii J. R., Baring M. G., 1998, ApJ, 509,238

[23] Jones, F. C., \& Ellison, D. C., 1991, Space Sci. Rev. , 58, 259,

[24] Kang H., Jones T. W., 2007, Astropart. Phys., , 28, 232

[25] Knerr, J. M., Jokipii, J. R. \& Ellison, D. C., 1996, ApJ, 458,641

[26] Klein, Karl-Ludwig \& Dalla, S. 2017, Space Sci. Rev. , 212, 1107

[27] Krymsky, G. F., 1977, Akad. Nauk SSSR Dokl., 243, 1306

[28] Lee, M.,1983, J. Geophys. Res., 88, 6109

[29] Lee, M.,2005, ApJS, 158, 38

[30] Li, G., Moore, R., Mewaldt, R. A., Zhao, L., \& Labrador, A. W. 2012, Space Sci. Rev. , 171, 141

[31] Li, G., \& Zank. 2005, in Proc. of 29th International Cosmic Ray Conference, Vol. 1, ed. B. Sripathi Acharya et al. (Mumbai: Tata Institute of Fundamental Research), 173

[32] Li, G., Zank, G. \& Rice, W., 2003, J. Geophys. Res., 108, 1082

[33] Li, G., Zank, G. \& Rice, W., 2005, J. Geophys. Res., 110, A06104

[34] Li, G. \& et al., 2009, ApJ, 702, 998

[35] Malkov, M. A., Drury L. O' C., 2001, Rep. Progr. Phys., , 64, 429

[36] Malkov, M. A., Diamond, P. H., \& Völk, H. J., 2000, ApJL533 , 171 119, 670

[37] Nitta, N. V., Liu, Y., DeRosa, M. L., \& Nightingale, R. W. , 2012, Space Sci. Rev. , 171, 61

[38] Ng, C., Reames, D., \& Tylka, A., 2003, ApJ, 591, 461

[39] Parker,E. N., 1961, Nucl. Energy, 2, 146

[40] Treumann, R. A., 2009, Astron. Astrophys., 17, 409-535

[41] Vladimirov, A., Ellison, D. C., \& Bykov, A., (2008), ApJ, 688, 1084 
[42] Wang, X. et al., 2013, ApJS, 209,18

[43] Wang, X. et al., 2011, Astron. Astrophys., 530, A92

[44] Wang, X. et al., 2012, Res. Astron. Astrophys., 12, 1535-1548

[45] Wang, X. et al., 2016, Res. Astron. Astrophys., 16, 32

[46] Wang, X. et al., 2017, ApJ, 842, 74

[47] Zank, G., Rice, W., \& Wu, C., 2000, J. Geophys. Res., 105, 25079

[48] Zirakashvili V. N., Aharonian F. A., 2010, ApJ, 708, 965 